\title{
Johanna Hoerning
}

\section{Städte zwischen »Eigenlogik « und Typik - eine Replik zur aktuellen stadtsoziologischen Debatte}

Gesellschaftliche Verhältnisse sind von Widersprüchen geprägt - da ist es keine Überraschung, dass wir das in sozialwissenschaftlichen Erklärungsansätzen wiederfinden. ${ }^{1}$ Die deutschsprachige Stadtsoziologie jedenfalls führt derzeit vor, wie Erklärungsansätze einander vehement widersprechen können. Mit meiner Replik auf die Debatte, wie sie in Heft 2/2013 dieser Zeitschrift zwischen Helmuth Berking als »Eigenlogiker « und Walter Siebel als Vertreter der » bisherige[n] soziologische[n] Stadtforschung « ${ }^{2}$ geführt wurde, verbinde ich hier vier Anliegen. Zum einen scheint mir, dass die Debatte kein Streit um konkurrierende Ansprüche in der Stadtforschung ist - zumindest nicht in einer Weise, dass sie sich gegenseitig ausschließen. Anstatt als Konkurrenzverhältnis lässt sich die Debatte auch als produktives Spannungsfeld begreifen. Dieses Spannungsfeld nährt sich aus verschiedenen wissenschaftstheoretischen Positionen ebenso wie aus den unterschiedlichen Reichweiten der Analyseperspektiven (1). Zum zweiten sollen zentrale Auslassungen beider Ansätze aufgezeigt werden. Dabei ist mein Einwand gegen Siebel, dass die von ihm vertretene "klassische" Perspektive der Stadtforschung ihrem Makro-Anspruch nicht gerecht werden kann, wenn sie sich auf » europäische « oder » westliche« Städte als (alleinigen) Gegenstand der Analyse beruft (2). In Bezug auf die »eigenlogische « Stadtforschung möchte ich zeigen, dass sie ihr kritisches Potenzial mindestens unausgeschöpft lässt, wenn sie dazu neigt, »Eigenlogik « als Essenz zu betonen (3). Entgegen jeder Essentialisierung stellt sich die Frage, wie (mögliche) »Eigenlogiken « produziert werden und wie sie sich in der Analyse rückbinden lassen an jene gesellschaftlichen Verhältnisse, innerhalb derer sie produziert und zum Ausdruck gebracht werden. Schließlich will ich (4) einen sehr kurzen theoretischen Ausblick wagen, wie die beiden Perspektiven sich in ein produktives Vermittlungsverhältnis bringen ließen - ohne sie darin auflösen zu wollen.

\section{Von Spannungsfeldern und Konfliktlinien}

»Ohne den Anspruch auf Totalität nehmen Praxis und Theorie das $>$ Wirkliche $<$ hin, wie es ist, sie akzeptieren die >Dinge<, wie sie sind: fragmentarisch, geteilt, vonein-

1 Einwenden ließe sich hier allerdings, dass es etwas sehr Verschiedenes ist, ob wir von Widersprüchen auf begrifflicher Ebene sprechen, welche durch Reflexion lösbar sein können, oder ob wir von Widersprüchen auf der Ebene der Praxis sprechen (vgl. die Unterscheidung von Widerspruchstypen bei Lefebvre 1987, S. 516 f.).

2 Siebel 2013, S. 248. 
ander abgesondert. «3 ${ }^{3}$ In Lefebvres Worten ist die »Wirklichkeit " zunächst » undurchdringlich «: Sie setzt der Analyse eine »augenblickliche Grenze ", aber eine, die das Denken als Herausforderung annehmen muss. Dabei wird Totalität einerseits methodologisch über die Idee und die Erkenntnis hergestellt, andererseits praktisch darüber, dass »[j]ede menschliche Tätigkeit, die in der gesellschaftlichen Praxis bestimmte Form angenommen hat [...] effektiv zur Totalität [tendiert] «. ${ }^{4}$ Diese grundsätzlichen Prämissen des Denkens lassen sich an beide hier diskutierten Ansätze der Stadtforschung herantragen. Damit geht es weder darum, die (städtische) Wirklichkeit als so differenziert hinzunehmen, wie sie sich uns im Alltag darstellt, noch sie als ganz und gar entfremdet und für theoretische Schlussfolgerungen eher hinderlich zu banalisieren.

Die Perspektive der »eigenlogischen Stadtforschung ", wie sie in Darmstadt entwickelt wurde, scheint vor allem eine Differenzlogik nahelegen zu wollen. Städte sind unterschiedlich, und diese Unterschiede werden zum paradigmatischen Ausgangspunkt einer »Soziologie der Städte ${ }^{5}$ gemacht. Dass Städte in dieser Sicht überhaupt miteinander vergleichbar sein können, hängt mit dem universellen Konzept von Stadt beziehungsweise dem Städtischen zusammen, das der (empirischen) Differenzlogik zugrunde liegt. ${ }^{6}$ Als Soziologie der Stadt kann demgegenüber die Position Walter Siebels gelesen werden, deren Aufgabe es ist, »das, was an Städten und ihren Entwicklungen und auch am einzelnen Fall einer bestimmten Stadt verallgemeinerbar ist ", $7 \mathrm{zu}$ erarbeiten.

Dabei impliziert die Perspektive der Eigenlogik offenbar, dass jede Stadt für sich genommen eine (möglicherweise beobachtbare) praktische Totalität darstellt. Untersucht werden kann dann, wie sich in jeder verorteten Praktik die Stadt als »Ganzes « dechiffrieren lässt, ein ihr inhärenter Code, der allem eingeschrieben ist. Die Stadt ist darin, so könnte man sagen, eine Welt »für sich « und lässt sich nicht reduzieren auf ein ihr äußeres Allgemeines. Der Speckgürtel Münchens, die hochpreisige Innenstadt und die randständigen residentiellen Gebiete von Menschen, die dem Image Münchens als einer reichen, »weißen « Stadt nicht entsprechen, würden dann in erster Linie auf eine spezifisch Münchnerische Art und Weise der Ein- und Ausgrenzung verweisen. Alle strukturellen Aspekte sind aus dieser Perspektive vermittelt über eine intrinsische Logik, und München verweist in erster Linie auf: München. Bei Walter Siebel scheint dagegen jede Stadt vorrangig auf eine dahinter

3 Lefebvre 1987, S. 437.

4 Ebd., S. $436 \mathrm{f}$.

5 Vgl. Löw 2008 (Hervorhebung J.H.).

6 Es basiert in Grundzügen auf der Wirth'schen Definition von Stadt als » relativ große, dicht besiedelte und dauerhafte Niederlassung gesellschaftlich heterogener Individuen " (Wirth 1974, S. 46.). Berking hebt dabei die »Prozesskategorien « (Berking 2013, S. 225) der Verdichtung und Heterogenisierung hervor und definiert die Stadt als »räumliches Strukturprinzip« (vgl. Berking 2008.).

7 Siebel 2013, S. 243 (Hervorhebung J.H.).

Leviathan, 42. Jg., 3/2014 
liegende Totalität - besser: auf ein dahinter liegendes Allgemeines - zu verweisen. ${ }^{8}$ An einer Stadt lässt sich dann zeigen, wie kapitalistische Logiken gesellschaftliche Widersprüche produzieren, die sich in einer Stadt (auch sichtbar) im Sinne von Spaltungen niederschlagen. Die oben beschriebenen Merkmale Münchens verweisen dann in erster Linie auf die gesellschaftlichen Verhältnisse von Ein- und Ausgrenzung, die sich in Suburbanisierung und Segregation in Städten niederschlägt.

Liegen hierin die zentralen Unterschiede zwischen den Positionen in der Debatte der deutschsprachigen soziologischen Stadtforschung? Wie lassen sich diese begreifen und ins Verhältnis zueinander setzen? Geht es nach Walter Siebel, dann stehen sich in dieser Debatte wissenschaftstheoretische Grundpositionen gegenüber. Ihm zufolge haben die beiden Ansätze je Gegenteiliges im Sinn: Der Eigenlogik-Ansatz verfolgt eine partikularisierende Stadtforschung, die auf einem für Siebel unzulässigen universellen Stadtbegriff aufbaut, worin ein idiographisches Erkenntnisinteresse zum Ausdruck gebracht werde. Dies klingt zunächst wie ein Widerspruch in sich. Dagegen setzt Siebel einen strikt historischen Stadtbegriff als Grundlage einer generalisierenden ${ }^{9}$ Stadtforschung entlang eines nomothetischen Erkenntnisinteresses. ${ }^{10}$ Diese Gegenüberstellung ist sehr aufschlussreich, zeigt sie doch, dass beide Ansätze in sehr unterschiedlicher Weise Logiken des Differenten und des Allgemeinen miteinander verbinden: Die Eigenlogik-These bezieht das Differente auf die empirische »Evidenz ", dass Städte unterschiedlich sind. Das Allgemeine ist dabei auf der Ebene der Konzepte verortet (die »Stadt" als räumliche Form). Für Siebel gelten Differenzierungen vorrangig im Hinblick auf Gesellschaftsspezifik und historisch. Innerhalb gesellschaftlich-geschichtlicher Formationen aber wird eine theoretisch begründete Verallgemeinerung konstatiert. Das Allgemeine bezieht sich hier eben nicht auf »das Städtische " (als universelles Konzept), sondern auf einen gesellschaftlich-geschichtlichen Typus, also etwa die »Europäische Stadt" oder die »Industriestadt «.

Mit Partikularisierung und Generalisierung sind nicht zuletzt zwei Pole vergleichender Forschung ${ }^{11}$ angesprochen, die weniger als gegensätzlich betrachtet, sondern vielmehr zueinander ins Verhältnis gesetzt werden sollten. Wie in anderen sozialwissenschaftlichen Forschungsgebieten auch ist damit in der Stadtforschung eine lange Debatte unterschiedlicher Positionierungen verbunden. ${ }^{12}$ Im Rahmen postkolonialer Forschungsbezüge ist in jüngerer Zeit (endlich wieder, möchte man

8 In jedem Fall kann es sich nur um partielle, nie um universelle Totalitäten handeln. Hier sei auf die Differenzierung von Totalität und partieller Totalität bei Lefebvre verwiesen.

9 Die Unterscheidung von »generalisierend « und » universell « erfolgt hier ganz bewusst: Eine über die einzelne (partikuläre) Stadt hinaus generalisierende Forschung, die vorrangig an Stadttypen interessiert ist, muss diese keinesfalls als universell geltend setzen, wie das Beispiel der »Europäischen Stadt « bei Walter Siebel offenkundig belegt.

10 Vgl. Siebel 2013, S. 240.

11 Vergleichende Forschung wird hier im umfassenden Sinn gebraucht, nicht in der eingeschränkten Version "gesellschafts-« oder »kulturvergleichender « Forschung.

12 Siehe hierzu etwa Abu-Lughod 1975; Abu-Lughod 2007; Masotti, Walton 1976; Simon 1988; Pickvance 1995; Ward 2010; Belina, Miggelbrink 2010; McFarlane 2010; Robinson 2011. 
sagen) verstärkt auf die methodologischen Aspekte diesbezüglich hingewiesen worden. Dabei sind pauschale Verallgemeinerungen in der »klassischen " Stadtforschung kritisiert worden, dass also Theoriebildung bis dato vorrangig auf europäischen und amerikanischen Stadtentwicklungen und deren Analyse basiert. Insbesondere aktuelle stadtgeographische Beiträge wie die von Jennifer Robinson und Colin McFarlane haben diesen Vorwurf vorgetragen. Ihre Kritik lautet, dass Stadtforschung (1) eine lineare (modernisierungstheoretische) Entwicklungsperspektive zum Ausdruck bringe (eine Stadt ist demnach »mehr « oder » weniger « entwickelt). Damit gehe einher, dass (2) Stadtvergleiche einer Logik des Ähnlichen folgten (wiederum gemessen am zugeordneten Entwicklungsniveau), was komplexitätsreduzierend wirke. Dabei werde immer wieder auf dieselben Städte zurückgegriffen, was unser Wissen über Städte auf einen kleinen Kreis »üblicher Verdächtiger « (von London über Berlin nach Chicago und Los Angeles) reduziere. Dahinter verberge sich nicht zuletzt auch (3) ein defizitäres Verständnis des Vergleichs, der auf die Relation zur prototypischen, paradigmatischen Stadt reduziert würde. ${ }^{13}$

Es liegt, so haben Ragin und Zaret einmal deutlich gemacht, in der Logik des Vergleichs (als grundlegender Form der Erkenntnisgewinnung), zwischen dem Allgemeinen und dem Besonderen zu unterscheiden. ${ }^{14}$ Wenn dem so ist, dann kann weder eine »Eigenlogik « von Städten noch ihre »Typik « absolut gesetzt werden, sondern dann müssen beide Aspekte zueinander ins Verhältnis gebracht werden. Zwar erkennen das beide hier diskutierten Ansätze an. Wenn aber Partikularisierung und Generalisierung tatsächlich eine trennscharfe Unterscheidung zwischen den beiden diskutierten Ansätzen zuließen, so müsste diese überwunden werden, und zwar beidseitig. Das ist bislang nicht zu erkennen. Denn wenn das Allgemeine in der paradigmatischen Setzung von Stadttypen liegt, die aus der Untersuchung westeuropäischer und nordamerikanischer Städte resultieren, dann werden Städte nur in ihrer Abweichung von der generellen Typik gedacht. Das macht aber nur Sinn, wenn man von einer allgemeinen, konvergenten oder linearen Stadtentwicklung ausgeht, die angesichts historisch und gesellschaftlich vielfältiger Differenzen wenig plausibel scheint. ${ }^{15}$ Ebenso lässt sich in Bezug auf die entgegengesetzte Perspektive formulieren: Wenn das Partikulare in der paradigmatischen Setzung der intrinsischen Logik einzelner Städte liegt, so würde damit die gesellschaftliche Produktion von Städten unberücksichtigt bleiben, und man liefe Gefahr, die für die Einordnung des Vorgefundenen relevanten Vergleichsmaßstäbe unbehelligt zu lassen.

Nun stellt sich bei jedem Vergleich unmittelbar die Frage nach dem Gegenstand des Vergleichens, womit nicht so sehr die explizite Definition von "Stadt " gemeint ist, sondern die theoretische Einordnung des Gegenstands. Denn zum einen wird "Stadt " in unterschiedlicher Weise sozial- beziehungsweise gesellschaftstheoretisch adressiert. Zum anderen scheint Stadt in je unterschiedlicher Weise als mikro- und makrosoziologisches Phänomen eingeordnet zu werden. Wenn man die Mikro-

13 McFarlane 2010, S. 728.

14 Ragin, Zaret 1983, S. 731.

15 Siehe hierzu auch Abu-Lughod 1975, S. 14.

Leviathan, 42. Jg., 3/2014 
Makro-Differenz als eine Frage der Reichweite ${ }^{16}$ bestimmt, dann zielt die Eigenlogik-Erklärung auf eine notwendig beschränkte Reichweite: Sie gilt eben nur für diese eine Stadt, die als ein Kontext sozialen Handelns identifiziert wird. Hier wird soziale Wirklichkeit lokal hergestellt, und lokal heißt dabei immer städtisch genauso wie lokalspezifisch. Auf der anderen Seite der Generalisierung findet sich dagegen der Anspruch, Städte als Makrophänomene zu verstehen, wobei ihr Allgemeines eben im Gesellschaftlichen, nicht im Städtischen liegt. Erklärungen werden dann nicht über Städte und Städtisches gesucht, sondern über den gesellschaftlichen Charakter von Stadtentwicklung.

Im Sinne von Partikularisierung und Generalisierung scheint »Stadt " als Gegenstand bei den hier besprochenen Ansätzen also ganz gegensätzlich zwischen den Polen des »Mikro-Makro-Spektrums « eingeordnet zu werden. Das verspricht unterschiedliche Einblicke in den Gegenstand. Beide Zugänge produzieren dabei allerdings blinde Flecken. Um Walter Siebel zu folgen: Es "geht um die Vermittlung dessen, was sich die beiden Ansätze als die jeweils vom anderen vergessene Seiten der Stadt als einer höchst widersprüchlichen Realität vorhalten «. ${ }^{17}$ Jeder der Ansätze hätte dementsprechend das jeweils »Vergessene « in die Reflexion mit einzubinden: einmal, um zu verhindern, dem Partikularen, Singulären und Differenten dessen, was sich uns als empirisch-evident aufdrängt, zu stark zu verfallen und damit die gesellschaftlichen Bedingungen seiner Genese unberücksichtigt zu lassen (Eigenlogik). Das andere Mal, um zu verhindern, dass die theoretischen Konzepte sich bei aller Generalisierung vollständig des Alltags entledigen. Wenn wir Städte gleichzeitig als Produkte/Ergebnisse und Bedingungen/Voraussetzungen des Alltags verstehen (und letzteren als beides: konkret-spezifisch wie auch allgemein), dann wird deutlich, dass sie eben beides sind: Mikro- wie Makrophänomen, Handlungskontext ebenso wie Ausdruck gesellschaftlicher Praxis.

Was die grundsätzliche Problematisierung des sozialtheoretischen Zugangs betrifft, ${ }^{18}$ so teile ich die Kritik am Eigenlogik-Ansatz nicht. Dass »Stadt « rein sozialtheoretisch bestimmt werden könnte, ist natürlich abwegig, schon allein deshalb, weil es sich dabei eben nicht nur um ein soziales Beziehungsgefüge beziehungsweise Verhältnis handelt. ${ }^{19}$ Aber »Stadt " ausschließlich gesellschaftstheoretisch zu bestimmen hieße nicht zuletzt, den Begriff der Praxis von den konkreten Praktiken in ihrem

16 So definiert bei Lindemann 2011, S. 5. Das ist interessant, weil damit impliziert scheint, dass immer die Vermittlung zwischen Individuum und Gesellschaft bearbeitet wird, egal ob nun die individuelle Handlungsebene (Mikro) oder gesellschaftliche Strukturen (Makro) untersucht werden. Entgegen diesem Gegenstandsbezug könnte man für die Unterscheidung von Sozial- und Gesellschaftstheorie anführen, dass hier jeweils die Erklärung für soziale Phänomene auf unterschiedlichen Ebenen gesucht wird: eben einmal auf der Ebene von sozialen Interaktionsverhältnissen, das andere Mal auf der von gesellschaftlichen Herrschaftsverhältnissen.

17 Siebel 2013, S. 259.

18 Kemper, Vogelpohl 2011, S. 17.

19 In den Worten Lefebvres ist die Stadt ein besonderes Werk (cuvre), das nicht nur eine konkret-materielle Faktizität setzt, sondern auch eine soziale Beziehungswirklichkeit zum Ausdruck bringt (Lefebvre 1996, S. 101 ff.). 
Handlungsbezug zu lösen. Dann ginge das Städtische im Gesellschaftlichen auf und ob die Menschen sich innerhalb der massiven gebauten Umwelt einer Stadt nun anders begegnen als auf einer Dorfstraße, könnte schnell für irrelevant erklärt werden. Die Vermittlung zwischen den Bedingungen gesellschaftlicher Produktion und Konsumption sowie den körperlich-dinghaften wie symbolisch-kulturellen Praktiken würde damit zu leicht aus dem Blick geraten. Wenn man Städte als besondere Orte begreift - und das tun Siebel und Berking gleichermaßen, wenn auch in unterschiedlicher Weise -, dann sind sie meines Erachtens auch als besondere Orte der (gesellschaftlichen) Praxis zu sehen, die als »Ort der Dialektik « ${ }^{20}$ von objektiven und subjektiven Strukturen konzipierbar ist.

\section{Von Kontexten und Kontextualisierungen - blinde Flecken einer strikt gesellschaftstheoretischen Stadtforschung?}

Wo liegen nun Grenzen der von Walter Siebel aufgezeigten gesellschaftstheoretischen Stadtforschung?21 Siebel führt in seiner Auseinandersetzung mit dem Eigenlogik-Ansatz zwei Aspekte ein, die er als Begründungszusammenhänge für eine "neue « Stadtforschung gelten lässt: die »verbesserte Theorie « sowie den »Wandel der Realität«.22 Diese können als zwei unterschiedliche Arten der Kontextualisierung von Stadtforschung verstanden werden - also einmal im Wissenschaftskontext, das andere Mal im Kontext des (historisch) reell Gegebenen. Beide Male muss aber gefragt werden, in welchem Rahmen das gilt. Für Siebel scheint diese Frage einfach zu klären: Es geht um »Stadtforschung im deutschen Sprachraum «, deren Gegenstand »weit überwiegend die europäische Stadt des 19. und 20. Jahrhunderts «23 sei. Aber hört der (relevante) Diskurs über Stadt an den Sprachgrenzen auf? ${ }^{24}$

Zentral erscheint mir hierbei allerdings weniger die sprachliche denn die regionale (»europäisch «) und historische (19./20. Jahrhundert) Abgrenzung des Gegenstands. Für meine diesbezüglichen Überlegungen beginne ich inhaltlich mit einer bewusst

20 Bourdieu 1993, S. 100.

$21 \mathrm{Zu}$ einer ausführlichen Diskussion des von Siebel und Häußermann vertretenen Ansatzes der Stadtsoziologie siehe auch die Debatte in sub\urban. zeitschrift für kritische stadtforschung, Heft 1/2013.

22 Siebel 2013, S. 242.

23 Ebd., S. 239.

24 Die Frage ist hier trotz aller notwendigen Abgrenzung des Untersuchungsfokus rhetorisch gemeint mit Blick auf die bereits geäußerte Kritik an der euro-nordamerikanischen Theoriegenerierung (nicht nur) in der Stadtforschung. Zudem gehört es heute ja zur guten wissenschaftlichen Praxis, Englischsprachiges zu rezipieren und produzieren. Bei der Fülle an Literatur, die dadurch für jede denkbare Forschungsfrage zu berücksichtigen ist, ist es selbstverständlich, dass jede Auseinandersetzung auf einer selektiven Forschungsrezeption beruht - der Vorwurf (gegenüber den EigenlogikerInnen) der Selektivität (ebd., S. 248) ist also pauschal und macht es sich zu einfach. Zumal pointierte Auseinandersetzungen mit einem Forschungsgegenstand notwendig selektiv sind, weil sie bestimmte Aspekte beleuchten und dafür in der Regel ihr Gegenteil hervorheben, um sich davor/ davon abzusetzen. 
pauschalen Aussage: Städte hat es zu ganz unterschiedlichen Zeiten, in sehr unterschiedlichen kulturellen und gesellschaftlichen Formationen gegeben, und sie sind zu einem Phänomen geworden, das sich sukzessive als vorrangige materielle Ausgestaltung menschlichen Zusammenlebens durchgesetzt hat. Warum ist diese Überlegung aber relevant? Welche Bedeutung, Funktion, Gestalt und Symbolik Städte jeweils ein- und annehmen, ist völlig different zu unterschiedlichen Zeiten, an unterschiedlichen Orten und im Rahmen verschiedener Gesellschaftsformationen. Insofern ist Siebel und dem von ihm zitierten Krämer-Badoni zuzustimmen, dass eine »Stadt ist, was eine historische [...] Gesellschaft sie zu sein bestimmt $" .{ }^{25}$ Die Aussage ist aber nur innerhalb eines allgemeineren begrifflich-theoretischen wie empirischgesellschaftlichen Zusammenhangs zu sehen. So hätte man die von Siebel als soziologisches Grundanliegen ausgewiesene Verallgemeinerbarkeit ${ }^{26}$ auch gegenüber der »Stadt « einzufordern: Die Konstanz des Phänomens müsste dann übersetzt werden in die übergreifende allgemeine Frage danach, wodurch sich das "Städtische " auszeichnet. Damit ist weniger eine abgeschlossene, einheitliche Bestimmung von "Stadt « gemeint - dafür variieren die jeweiligen Ausprägungen und inhaltlichen Bestimmungen historisch und gesellschaftsspezifisch mit Sicherheit zu sehr -, sondern allgemeine Begrifflichkeiten, die das Besondere des "Städtischen« analytisch $\mathrm{zu}$ fassen suchen.

Um zu verdeutlichen, welche Dimension diese Herausforderung hat, greife ich ein klassisches Beispiel auf: Reinhard Bendix hat 1963 einige Überlegungen zum Problem der Übertragbarkeit von Konzepten im Kontext vergleichender Forschung am Begriff der »Urbanität « ${ }^{27}$ angestellt. ${ }^{28}$ Das Konzept stelle auf abstrakte Weise dar, was wir über westliche Städte in Erfahrung gebracht haben, könne aber keine universelle Geltung beanspruchen. Bendix meint dabei allerdings die spezifische Ausprägung von "Urbanität ", wie sie von Simmel und im Anschluss daran auch von Wirth analysiert wurde. Damit sind bestimmte Qualitäten von »Urbanität « bezeichnet, wie sie für moderne Großstädte in Europa und auch Nordamerika Geltung beanspruchen können/konnten und die in der Folge häufig normativ gesetzt wurden. Es sind aber nicht die spezifischen Qualitäten der »Urbanität als Lebensform " (»urbanism as a way of life) ", die das analytische Konzept dieses Begriffs von »Urbanität « begründen. Vielmehr werden auf analytischer Ebene die strukturellen Determinanten Größe, Dichte und Heterogenität (nach Wirth) für Städte geltend gemacht, die in Verbindung mit spezifischen Normen, Werten etc. zur Herausbildung distinkter Lebensweisen führen. ${ }^{29}$ Folgt man Bendix, müsste genau dieses

25 Krämer-Badoni 1992, S. 27, so zitiert in Siebel 2013, S. 239.

26 Siebel 2013, S. 243.

27 Der Urbanitäts-Begriff ist hier deshalb in Anführungsstriche gesetzt, weil die Übersetzung von »urbanism as a way of life " (Wirth) als »Urbanität als Lebensform « zwar geläufig, aber nur sehr ungenau ist.

28 Bendix 1963, S. $534 \mathrm{f}$.

29 Diese unterscheiden sich annahmegemäß von Lebensformen in nicht oder weniger städtischen Gegebenheiten/Orten - Kontexten also, die weniger dicht, groß und heterogen wären. 
analytische Konzept universelle Geltung beanspruchen. Zwar könnten sich die Ausprägungen unterscheiden, der Grundgedanke aber, dass sich durch die spezifisch städtischen Bedingungen von anderen Orten differente Lebensweisen herausbilden, hätte universelle Geltung zu beanspruchen. Diesem Anspruch folgt, wie es scheint, der Ansatz der Eigenlogik-Forschung. Nun gibt es keine universelle Kausalität zwischen Stadt, städtischer Lebensweise und den spezifischen Erscheinungen von Anomie, Individualismus, Kosmopolitanismus, Rationalität, Blasiertheit etc. Untersuchen lässt sich jedoch die These (!) einer konzeptuellen Ursächlichkeit, welche strukturell-räumliche Merkmale mit sozialen Organisationsformen in Verbindung bringt. ${ }^{30}$ Dafür braucht es keine universell gültige (inhaltliche) Bestimmung von Stadt. Gleichzeitig ist eine gesellschaftstheoretische Reflexion der strukturell-räumlichen Merkmale gerade hier wohl vonnöten, um nicht in die sozialökologische Falle zu tappen, Soziales mit Nicht-Sozialem zu erklären. Letztlich heißt das, die räumlich-strukturellen Merkmale nicht als gesetzte Bedingungen zu verstehen, sondern ihre gesellschaftliche Bedingtheit selbst mit einzubeziehen.

Die reservierte Haltung Siebels gegenüber der konzeptuellen Universalität, also der Behauptung eines universell gültigen (analytischen) Stadtbegriffs, ist ja auch keinesfalls unbegründet: Die Aussagen, welche wir über Städte treffen können, sind notwendig kontextgebunden. Aber gerade um das Kontextgebundene daran zu verstehen, sind darüber hinausgehende Vergleiche von elementarer Bedeutung. $\mathrm{Zu}$ schnell führt eine Reduktion der (prinzipiell an Generalisierung interessierten) Forschung dazu, dass einzelne Konzepte absolut gesetzt werden und Inhalte mit dem analytisch Allgemeinen verwechselt werden, die dann als normative Setzungen fungieren (wie etwa beim Urbanitätsbegriff). An Colin McFarlanes (und Jennifer Robinsons) Kritik der Vergleichsmethodologie »klassischer" Stadtforschung anschließend, ist auch hier eine Reduktion auf »europäische Städte« in Bezug auf eine historische und gesellschaftsspezifische Einschränkung wenig zielführend, denn erstens ist fraglich, welcher gemeinsame Gesellschaftskontext hierfür den Rahmen stellt. ${ }^{31}$ Zweitens steht in Frage, ob die »europäische Stadt « ohne ihr Anderes verstanden werden kann. Was ist damit gemeint? Der Typus suggeriert eine Spezifik, und die historische Analyse dieses Typs zeigt dessen historische Spezifik im Rahmen der eigenen Entwicklungsgeschichte, nicht aber, in welcher Relation diese Entwicklungsgeschichte steht. Dabei ist auch fraglich, inwiefern die historische Spezifik abgrenzbar ist: So ist etwa die Idee der emanzipatorischen Stadt ${ }^{32}$ als ein zentrales Merkmal des »Europäischen Stadttypus « nicht loszulösen von der zeitgleichen Pro-

30 Der Kritik Siebels, dass eine Verabsolutierung dieser » räumlich-strukturellen « Merkmale kein soziologisch relevanter Zugang ist, ist zwar prinzipiell zuzustimmen. Die räumliche Bestimmung von Stadt ist aber meines Erachtens nicht gleichzusetzen mit einer banalen Raumauffassung, und die völlige Abwendung von räumlich-strukturellen Bestimmungsmerkmalen ist wenig erquicklich (so bei Häußermann, Siebel 1974), zumindest wenn man als raumtheoretische Grundannahme geltend macht, dass der (soziale) Raum ein (soziales) Produkt ist (vgl. Lefebvre 2001 [1991], S. 26).

31 Es müsste sich ja um einen »europäischen Gesellschaftskontext « handeln, der aber unbegründet bleibt.

32 Siebel 2004, S. 13.

Leviathan, 42. Jg., 3/2014 
duktion extremer Unterdrückung in europäischen Städten selbst genauso wie in "nicht-europäischen Städten " in kolonialen Kontexten. Dass das europäische Modell eines industriellen, verstädterten Kapitalismus sich dabei unter anderem eben gerade auf der Grundlage kolonialer Ausbeutungskontexte durchsetzen konnte, ist dabei nicht unwesentlich. Die Reichweite im Hinblick auf eine Einbettung soziologischer Stadtforschung in makrosoziologische Gesellschaftstheorie ist daher meines Erachtens nicht zu erreichen, wenn man die Erkenntnisse über deutsche, europäische oder nordamerikanische Städte nicht ihrerseits ins Verhältnis setzt zu Städten, die sich unter in vielerlei Hinsicht sehr andersartigen Bedingungen entwickeln. Auch hier müsste es sowohl um das Herausarbeiten von Spezifika und lokalen Besonderheiten einerseits und von Typik und generalisierbaren Aussagen andererseits gehen.

Dem Verständnis der verwobenen Geschichten ${ }^{33}$ nach müsste auch Stadtentwicklung aus dem gemeinhin angenommenen »Kontext « euro-nordamerikanischer (»westlicher «) Verhältnisse gelöst und in einen weiteren Kontext gesetzt werden, der auf die vielfältigen Brüche und Kontinuitäten von Machtstrukturen verweist, welche städtische genauso wie ökonomische, politische und gesellschaftliche Entwicklungen prägen. Das Andere, ohne das die europäische Stadt nicht verstanden werden kann, ist selbst aber keine feste Größe. Die Grenze verläuft und verschiebt sich beständig innerhalb der unterschiedlichen Typologien von Städten, etwa von der "amerikanischen « über die »(post)koloniale» bis hin zur »Megastadt « - die nicht zuletzt zusammenfallen können, aber je sehr Unterschiedliches meinen und die Stadt in verschiedene Verhältnisse setzen (regionale, polit-ökonomische, Größenverhältnisse und andere). Aber auch jede Typenbestimmung zieht neue Grenzen und ist als solche in sich nicht konstant (etwa bei der Größenbestimmung angesichts sich verändernder Größenverhältnisse). Und schließlich verschieben sich auch die Grenzziehungen und Ausgrenzungen innerhalb der »europäischen Städte "selbst, wo die Regulierung des Anderen etwa übersetzt wird in die Kontrolle von Körpern von MigrantInnen und Flüchtlingen. ${ }^{34}$

Wenn das so ist, dann kann es weder »nur « eine historisch-gesellschaftsspezifische noch » nur « eine universell-eigenlogische Stadtforschung geben. Mir scheint, es ist weniger die Frage nach der Eigenlogik, die zur Debatte steht, sondern das konkrete Verständnis von Eigenlogik: Dann ginge es vor allem darum, sie nicht als eine substanzielle (wenn auch mentale) "Gestalt"von Städten zu verstehen, sondern die Prozesse zu untersuchen, welche eine solche »kumulative Textur " (Berking) herstellen.

33 Conrad, Randeria 2002.

34 Siehe hierzu Ha 2014. 


\section{Kritisches Potenzial der Eigenlogik-Forschung?}

Wie steht es also um die Grenzen der Perspektive der Eigenlogik von Städten?35 Angenommen, städtische Entwicklungen und damit auch die Richtung, welche Konflikte und Auseinandersetzungen um städtische Räume annehmen (können), folgten tatsächlich einer eigenlogischen Struktur, so läge im Eigenlogik-Ansatz auch ein Potenzial für (emanzipatorische Gegenwarts-)Kritik. ${ }^{36}$ Denn geht man davon aus, dass es bestimmte (eigenlogische) Narrative oder Bewertungs- und Handlungsmuster gibt, welcher sich die Akteure, ob intentional oder nicht, in der Austragung von Interessenskonflikten bedienen, so wäre die Kenntnis darüber Voraussetzung für ihre Überwindung. Das heißt, wenn etwa die (eine?) Eigenlogik Berlins darin bestehen sollte, Armut und Ungleichheit zur Ressource von »Kreativität mit einem Schuss Exzentrik, Jugendlichkeit und Lebensfreude ${ }^{37}$ zu stilisieren, so wäre die wissenschaftliche Aufarbeitung dieser »kumulativen Textur « in mehrfacher Hinsicht von Bedeutung: zum einen, weil sie als Grundlage für eine kritische Diskursanalyse der machtvollen politischen und ökonomischen Akteure dienen könnte. Zum anderen, weil sie es ermöglichen würde, das darin liegende Entfremdungspotenzial kritisch zu reflektieren im Hinblick auf den Alltag der Menschen. Denn wenn Armut und Ungleichheit in institutionalisierter Weise (und zwar ganz allgemein »in Berlin«) zur produktiven Ressource banalisiert würden, dann hätte das extrem negative Konsequenzen für die Ermöglichung und Legitimierung von Kritik an diesen Verhältnissen. Wenn eine Romantisierung schwieriger materieller Existenzbedingungen habituell inkorporiert würde, so läge in einem solchen eigenlogischen Narrativ oder Muster eine enorme herrschaftsstabilisierende Kraft.

In der wissenschaftlichen Benennung eigenlogischer Städtedynamiken liegt damit ein gleichermaßen affirmatives wie kritisches Potenzial. Das Potenzial der Eigenlogik-Forschung könnte also gerade in der Kritik der Eigenlogik liegen und damit in einer Reflexion der unhinterfragt reproduzierten Denk- und Handlungsschemata. Sie zu identifizieren wäre insofern (nur) ein erster, wichtiger Schritt. Die Frage nach lokaler Spezifik ist relevant. Der Eigenlogik-Ansatz bindet diese Perspektive soziologisch ein in die Frage nach einer lokalstrukturellen Logik, die dauerhaft in den sozialen Kontexten lokalen Alltagslebens Wirkung und Bedeutung entfaltet. In jedem Fall sollte aber auch hier das eingefordert werden, was Berking für die Stadt als » raumstrukturelle Form " schon postuliert hat: dass es sich um eine grundlegend dynamische Form handelt, der mit Verdichtung und Heterogenisierung »Prozesskategorien « zugrunde liegen. Es kann dann nicht darum gehen, etwas zu unter-

35 Zugunsten der Frage nach einem kritischen Potenzial eigenlogischer Stadtforschung wird hier auf eine detaillierte Auseinandersetzung mit dem Ansatz verzichtet. Verwiesen sei auf die umfangreiche, kritische Debatte in einem von Jan Kemper und Anne Vogelpohl herausgegebenen Sammelband (Kemper, Vogelpohl 2011).

36 Verwiesen sei hier auf die Begriffsklärungen in Jaeggi, Wesche 2013 [2009], S. 10 f., wenngleich ich hier auf theoretische Klärungen zum Kritikbegriff verzichte. Stattdessen hoffe ich, dass im folgenden empirischen Bezug klar wird, was für ein Verständnis damit impliziert ist.

37 Löw 2008, S. 201.

Leviathan, 42. Jg., 3/2014 
suchen, was als ontologische Entität (oder eigenlogische »Essenz «) von Stadt begriffen werden kann. Stattdessen handelt es sich wohl um ein beständiges Nebeneinander von Prozessen, die »das Ganze der Stadt « gleichzeitig herstellen wie nicht herstellen.

Möglicherweise ist es aber weniger relevant, ob es so etwas wie stadtspezifische Symboliken gibt, die im Alltag als Referenz dienen. Die These der Eigenlogik ist als Alltagserfahrung ja ebenso einleuchtend (in Berlin ist man eben »berlinerisch ", in Frankfurt »frankfurterisch «) wie nicht einleuchtend (wie soll eine alleinerziehende Mutter und Hartz IV-Empfängerin aus Marzahn oder Bonames dieselbe Vorstellung von Berlin oder Frankfurt haben wie ein hochbezahlter Unternehmensberater und Single mit Wohnsitz in einem innenstädtischen Townhouse?). Theoretisch-abstrakt entfaltet diese These wenig Schlagkraft, empirisch begründet könnte sie aber eine eindrucksvolle Referenz für Stadttheorie darstellen. Theoretisch vielversprechender scheint mir deshalb die Frage danach, auf welche Weise diese symbolischen Referenzen handlungsrelevant werden (und das nicht nur auf diskursiver Ebene, sondern auch im Sinne körperlich-materieller Praktiken), wodurch es auch möglich wäre, zu berücksichtigen, inwiefern sie machtvermittelt sind. Dann würde wiederum die Vermittlung zwischen dem Lokal- oder Stadtspezifischen und den bedingenden gesellschaftlichen Strukturen in den Vordergrund rücken. In den sozialen Praktiken würde dann nicht nur »Gesellschaft « beziehungsweise gesellschaftliche Praxis hergestellt, sondern auch (eine) »Stadt « beziehungsweise (eine) städtische Praxis.

\section{Städte zwischen Homogenisierung und Heterogenisierung}

Möglicherweise gibt es eine produktive Schnittmenge zwischen den beiden Ansätzen der Forschung in Städten und der Forschung über Städte, die sich wie folgt formulieren lässt: Städte sind gleichermaßen gesellschaftliche wie soziale Verhältnisse; sie sind Handlungskontexte ebenso wie Ausdruck gesellschaftlicher Praxis. Dabei bringen sie Gesellschaft nicht einfach nur zum Ausdruck, genauso wenig wie das Lokale schlichte Manifestation globaler Verhältnisse ist. Die beiden hier diskutierten Zugänge schließen sich also nicht aus, sondern es geht, wie Siebel auch vermerkt, um deren Vermittlungsverhältnis im Sinne eines Verständnisses von Städten als »höchst widersprüchlichen Realität[en] «. ${ }^{38}$ In der Ausformulierung dessen, was das bedeutet, liegt vermutlich eine zentrale Aufgabe der Stadtforschung, die ich hier nur kurz und grob skizzieren kann.

Die beiden Ansätze betonen je spezifische Aspekte des Städtischen und legen dabei einmal die Betonung auf das, was Städte als Einheiten konstituiert, und das andere Mal darauf, was sie zum Teil einer übergeordneten Einheit (Gesellschaft) macht. "Die Stadt « als Einheit ist eine Abstraktion, sie ist der Fluchtpunkt des Denkens, schafft eine Abgrenzung und verweist auf die in sich un-einheitliche Wirklichkeit. Das Gleiche gilt ja auch für Gesellschaft: Eine Setzung von Gesellschaft als geschlossenes »Objekt« suggerierte bloß »einen Zusammenhalt, den sie [die Gesellschaft] 
gänzlich vermissen lässt angesichts einer Totalität, die tatsächlich dezidiert offen ist - so offen sogar, dass sie auf Gewalt gründen muss, um fortzubestehen «. ${ }^{39}$ Gleichzeitig und anders als »die Gesellschaft « hat »die Stadt « - und insofern ist der Begriff kein genuin soziologischer - auch eine physisch-materielle »Gestalt ", eine sichtbare Form, eine Dinglichkeit, die Abgeschlossenheit suggeriert. So wenig wie Städte auf diese physische Substanz reduziert werden können, so wenig können sie »nur « als mentale Gestalt verstanden werden. Die Stadt ist insofern ein »sozialer Raum ", der als »soziales Produkt « zu verstehen ist, welches konkrete Form annimmt. ${ }^{40}$ Versteht man die Stadt beziehungsweise die Städte als gesellschaftliche Verhältnisse in ihrer Widersprüchlichkeit, dann rückt nicht eine Seite von, sondern die (häufig konflikthafte) Simultaneität selbst in den Blick, in der soziale und gesellschaftliche Homogenisierung und Heterogenisierung, Ein- und Ausgrenzungen, Differenzen und Identitäten produziert werden. So verstanden, als produzierter städtischer Raum im Lefebvre'schen Sinn, rücken die Aushandlungen über die simultane praktische Herstellung von Homogenisierungen und Heterogenisierungen auf der materiellen, symbolischen wie konzeptuell-diskursiven Ebene in den Blick. In dieser simultanen Widersprüchlichkeit wird die (Idee der) Stadt als »Ganzes « ebenso hergestellt, wie sie darin beständig infrage gestellt und aufgelöst wird. Das zu denken und zu rekonstruieren könnte Aufgabe einer Stadtforschung sein, die nicht Aspekte des Städtischen untersuchen will, sondern nach der gesellschaftlichen Produktion des Städtischen fragt.

\section{Literatur}

Abu-Lughod, Janet 1975. »The legitimacy of comparisons in comparative urban studies. A theoretical position and an application to North African cities ", in Urban Affairs Review 11, 1, S. 13-35.

Abu-Lughod, Janet 2007. »The challenge of comparative case studies «, in City 11, 3, S. 399-404.

Belina, Bernd; Miggelbrink, Judith. Hrsg. 2010. Hier so, dort anders. Raumbezogene Vergleiche in der Wissenschaft und anderswo. Münster: Westfälisches Dampfboot.

Bendix, Reinhard 1963. "Concepts and generalizations in comparative sociological studies ", in American Sociological Review 28, 4, S. 532-539.

Berking, Helmuth 2008. " Städte lassen sich an ihrem Gang erkennen wie Menschen< - Skizzen zur Erforschung der Stadt und der Städte ", in Die Eigenlogik der Städte. Neue Wege für die Stadtforschung, hrsg. v. Berking, Helmuth; Löw, Martina, S. 15-32. Frankfurt a. M.: Campus.

Berking, Helmuth 2013. »StadtGesellschaft. Zur Kontroverse um die Eigenlogik der Städte«, in Leviathan 41, 2, S. 224-237.

Bourdieu, Pierre 1993. Sozialer Sinn. Kritik der theoretischen Vernunft. Frankfurt a. M: Suhrkamp.

Conrad, Sebastian; Randeria, Shalini 2002. »Geteilte Geschichten - Europa in einer postkolonialen Welt ", in Jenseits des Eurozentrismus. Postkoloniale Perspektiven in den Geschichtsund Kulturwissenschaften, hrsg. v. Conrad, Sebastian; Randeria, Shalini, S. 9-49. Frankfurt a. M.: Campus.

Ha, Noa 2014. »Perspektiven urbaner Dekolonisierung: Die europäische Stadt als >Contact Zone«", in sublurban. zeitschrift für kritische stadtforschung 1, S. 27-48.

39 Lefebvre 2001 [1991], S. 11 (Übersetzung J.H.).

40 Vgl. den Raumbegriff Lefebvres (siehe oben).

Leviathan, 42. Jg., 3/2014 
Häußermann, Hartmut; Siebel Walter 1974. »Thesen zur Soziologie der Stadt ", in Leviathan 6, S. 484-500.

Jaeggi, Rahel; Wesche, Tilo 2013 [2009]. „Einführung: Was ist Kritik?", in Was ist Kritik? Philosophische Positionen, hrsg. v. Jaeggi, Rahel; Wesche, Tilo, S. 7-22. 3. Aufl. Frankfurt a. M.: Suhrkamp.

Kemper, Jan; Vogelpohl, Anne 2011. » Eigenlogik der Städte<? Kritische Anmerkungen zu einer Forschungsperspektive", in Lokalistische Stadtforschung, kulturalisierte Städte. Zur Kritik einer »Eigenlogik der Städte", hrsg. v. Kemper, Jan; Vogelpohl, Anne, S. 15-38. Münster: Westfälisches Dampfboot.

Lefebvre, Henri 1987. Kritik des Alltagslebens. Grundrisse einer Soziologie der Alltäglichkeit. Frankfurt a. M.: Fischer.

Lefebvre, Henri 1996. Writings on cities, hrsg. v. Kofman, Eleonore; Lebas, Elizabeth. Oxford, Cambridge: Blackwell.

Lefebvre, Henri 2001 [1991]. The production of space. Reprinted. Oxford: Blackwell.

Lindemann, Gesa 2011. »Die Gesellschaftstheorie von der Sozialtheorie her denken - oder umgekehrt? ", in ZfS-FORUM 3, 1, S. 1-19.

Löw, Martina 2008. Soziologie der Städte. Frankfurt a. M.: Suhrkamp.

Masotti, Louis; Walton, John 1976. »Comparative urban research. The logic of comparisons and the nature of urbanism ", in The city in comparative perspective. Cross-national research and new directions in theory, hrsg. v. Walton, John; Masotti, Louis, S. 1-15. New York: John Wiley \& Sons.

McFarlane, Colin 2010. »The comparative city: knowledge, learning, urbanism «, in International Journal of Urban and Regional Research 34, 4, S. 725-742.

Pickvance, Chris 1995. »Comparative analysis, causality and case studies in urban studies ", in The urban context. Ethnicity, social networks, and situational analysis, hrsg. v. Rogers, Alisdair; Vertovec, Steven, S. 35-54. Oxford: Berg.

Ragin, Charles C.; Zaret, David 1983. "Theory and method in comparative research. Two strategies ", in Social Forces 61, 3, S. 731-754.

Robinson, Jennifer 2011. "Cities in a world of cities: the comparative gesture ", in International Journal of Urban and Regional Research 35, 1, S. 1-23.

Siebel, Walter 2004. »Einleitung: Die europäische Stadt «, in Die europäische Stadt, hrsg. v. Siebel, Walter, S. 11-50. Frankfurt a. M.: Suhrkamp.

Siebel, Walter 2013. »Stadt, Ungleichheit und Diversität", in Leviathan 41, 2, S. 238-263.

Simon, Karl-Heinz 1988. »Probleme vergleichender Stadtforschung ", in Soziologische Stadtforschung, hrsg. v. Friedrichs, Jürgen, S. 381-409. Opladen: Westdeutscher Verlag.

Ward, Kevin 2010. "Towards a relational comparative approach to the study of cities ", in Progress in Human Geography 34, 4, S. 471-487.

Wirth, Louis 1974. "Urbanität als Lebensform«, in Stadt-und Sozialstruktur. Arbeiten zur sozialen Segregation, Ghettobildung und Stadtplanung, hrsg. v. Herlyn, Ulfert, S. 42-66. München: Nymphenburger. 
Zusammenfassung: Die Replik greift die aktuelle Debatte zwischen zwei differierenden Ansätzen der deutschsprachigen Stadtsoziologie auf, wie sie von Helmuth Berking und Walter Siebel formuliert werden. Dabei wird die These vertreten, dass es sich nicht um widersprüchliche Ansätze handelt, sondern dass vielmehr das Städtische als ihr Gegenstand von einem Widerspruch geprägt ist, der sich als Simultaneität von Homogenisierung und Heterogenisierung begreifen lässt.

Stichworte: Stadtsoziologie, »Eigenlogik« der Städte, Debatte

\title{
Cities between »intrinsic logic « and typology - a reply to the current debate on urban sociology
}

Summary: The reply deals with the current debate between the two differing approaches to German urban sociology as proposed by Helmuth Berking and Walter Siebel. It is argued that they are not to be understood as contradictory approaches. Instead, it is rather their object of study - the urban - that is contradictory in terms of its simultaneity of homogenization and heterogenization.

Keywords: urban sociology, »intrinsic logic « of cities, debate

\author{
Autorin \\ Johanna Hoerning \\ Technische Universität Berlin \\ FH 9-1 \\ Fraunhoferstr. 33-36 \\ 10587 Berlin \\ johanna.hoerning@tu-berlin.de
}

